\title{
TWO-SENSOR NEURAL NETWORK MODELING FOR FAULT DETECTION
}

\author{
Robert E. Uhrig", Israel Alguindigue, Anna Loskewicz-Buczak, \\ Andreas Ikonomopoulos, and Lefteri, Tsoukalas* \\ University of Tennessee, Knoxville, TN 37996-2300 \\ - Also at Oak Ridge National Laboratory, Oak Ridge, TN 37831-6005 \\ - Now with Japan Atomic Energy Research Institute, Tokai-Mura, Japan
}

\begin{abstract}
The technique described here involves training a neural network to model the internal behavior of a component or system from vibration data taken from two sensors (accelerometers) located at different positions or mounted in different directions on the component or system. The power spectral density (PSD) of the sampled time-series from one accelerometer is used as the input to the neural network, and the PSD of the sampled time-series from the other accelerometer is the desired output of the neural network. The network is trained while the signals varies over the normal range of operation when the component or system is known to be operating properly. The trained neural network is then used in a monitoring mode to predict the output sensor PSD from the input PSD, and a comparison is made between the predicted and actual output PSDs. Significant deviations indicate that the interrelationship between the input and output signals has been modified due to a change (failure) in the component or system. The usefulness of this methodology has been demonstrated in the monitoring of the operability of check valves and a pump-motor bearing.
\end{abstract}

Check Valve Monitoring. The most common problems associated with check valve failures are due to system flow oscillations or system piping vibrations. These vibrations and oscillations induce measurable accelerations that produce check valve component wear and thus component failure. Analysis of time-records from piezoelectric accelerometers attached to check valves on a large nuclear power plant has been used to demonstrate this process. An autoassociative-like neural network in which the inputs and desired outputs are values of the PSDs of two related time-series representing vibration at two different positions on the valve was trained to produce a neural network model of the interrelationship when the valve is operating properly. During monitoring, the output PSD of one accelerometer is used to predict the output PSD of the other accelerometer. This predicted PSD is then compared with the actual PSD, and a significant deviation indicates failure of the check valve. Comparison of PSD spectra between identical 30-inch check valves (one broken and one normal), operating under identical conditions, demonstrated that this technique can identify the failed valve. Subsequent measurements taken on the broken valve after it was repaired further confirmed the validity of this technique.

Analysis of Vibrations. The two-sensor technique was also used to analyze the progressive failure of a large (950 HP) motor-pump bearing in a nuclear power plant. A series of measurements of horizontal and vertical components of acceleration for the motor-pump bearing were taken periodically throughout the operating lifetime of the bearing and as it began to fail. The PSDs of the horizontal and vertical components of acceleration on the bearing during the first four sets of measurements (when the bearing was known to be operating properly) were the input and desired output respectively of a neural network while it was being trained. For the next eight sets of measurements, while the bearing progressed towards failure, the predicted value of the vertical component of acceleration (obtained from the neural network using the horizontal component as the input) was compared with the actual value of the vertical component. The deviation between the predicted and actual vertical PSDs grew as the bearing progressed towards failure. 